\title{
Peripheral T-Cell Lymphomas-Not Otherwise Specified: A Shrinking Entity?
}

Presented by Steven M. Horwitz, MD

\section{ABSTRACT}

Peripheral T-cell lymphomas-not otherwise specified (PTCL-NOS) is a broad category of biologically and clinically heterogeneous diseases, which likely does not have a single treatment paradigm. Understanding of subtype-specific approaches is leading to more individualized therapy. There are also therapeutic vulnerabilities to target, such as CD30, JAK/STAT pathway, and epigenetic modifiers, that may cross different histologic subtypes. As new therapies evolve, however, it is important to understand in which situations current standard treatments work, because some of these treatments, such as combination chemotherapy, are potentially curative for a subset of patients. For certain populations, adding to these chemotherapy backbones will produce the best results. For other populations, entirely new approaches may be appropriate. Future treatment advances will, in part, be made by enriching populations based on their likelihood of response to specific therapies and utilizing biomarker-driven or biomarker-informed strategies.

J Natl Compr Canc Netw 2020:18(12.5):1767-1769 doi: 10.6004/jnccn.2020.5037

Peripheral T-cell lymphomas-not otherwise specified (PTCL-NOS) has become a shrinking entity as clinically meaningful subtypes are increasingly recognized and subtype-specific therapies are approved. At the NCCN 2020 Virtual Congress: Hematologic Malignancies, Steven M. Horwitz, MD, Associate Attending Physician, Memorial Sloan Kettering Cancer Center, and Chair of the NCCN T-Cell and Primary Cutaneous Lymphomas Panels, discussed efforts to build on standard-of-care chemotherapy and move toward more individualized treatment.

As Dr. Horwitz explained, compared with diffuse large B-cell lymphoma (DLBCL), PTCL has a poorer prognosis, with more patients experiencing disease relapse, having disease that fails to respond to therapy, and ultimately dying from the disease. However, one patient population that stands out are those with $A L K$-positive anaplastic large cell lymphoma (ALCL). This favorable subgroup exhibits higher cure rates than most patients treated with standard chemotherapy regimens such as CHOP (cyclophosphamide/doxorubicin/vincristine/ prednisone).

Although there are nearly 30 different subtypes of PTCL, the 3 most common subtypes comprise approximately two-thirds of patients: PTCL-NOS, angioimmunoblastic T-cell lymphoma (AITL), and one of the varieties of systemic ALCL.

\section{Dose Intensification}

According to Dr. Horwitz, an increased understanding of the biology of different subtypes of T-cell lymphoma is currently leading to more individualized therapy.
Historically, multiple attempts to improve on the standard regimen of CHOP have been unsuccessful. The one strategy, however, that may have benefit in standard practice is dose intensification with etoposide and autologous stem cell transplantation (autoSCT).

A number of studies from the German High-Grade Non-Hodgkin Lymphoma Study Group, conducted before rituximab became standard therapy for aggressive B-cell lymphoma, looked at the addition of etoposide to CHOP. ${ }^{1}$ Although most patients had B-cell lymphoma, a subgroup analysis of patients with T-cell lymphoma showed that the addition of etoposide improved event-free survival. However, most of the benefit was derived from patients with $A L K$ positive ALCL, said Dr. Horwitz. When those patients were removed, there was only a trend in benefit in terms of event-free survival and no benefit in overall survival (OS).

More dose intensity involves consolidating patients with autoSCT. To evaluate the efficacy of a dose-dense approach consolidated by up-front high-dose chemotherapy and autoSCT in PTCL, the Nordic Lymphoma Group conducted a large prospective phase II study in untreated systemic PTCL. ${ }^{2}$ Most patients experienced a response to treatment, with an overall response rate of $82 \%$, a complete response rate of $51 \%$, and a 5 -year progression-free survival (PFS) rate of $44 \%$. When analyzed according to subtype, those with $A L K$-negative ALCL seemed to perform the best, although PFS for other more common subtypes of PTCL-NOS and AITL were 
approximately $40 \%$. "These are phase II data, and there is some selection bias because it's a transplant study, but these results may be better than what we would see historically with CHOP alone," stated Dr. Horwitz, who noted that he has adopted this approach as a no-protocol standard for patients with PTCL. "We do note, however, that this treatment does come with some increased toxicity, especially hematologic toxicity."

\section{Brentuximab Vedotin}

According to Dr. Horwitz, the biggest advance in the treatment of PTCL has come with the addition of brentuximab vedotin (BV). This is based on encouraging activity observed in the phase III ECHELON-2 trial, which compared the efficacy and safety of $\mathrm{BV}+$ cyclophosphamide/doxorubicin/prednisone (CHP) versus CHOP for the treatment of CD30-positive PTCL. ${ }^{3}$ The double-blind, double-dummy, randomized, placebocontrolled, active-comparator phase III study enrolled 452 patients. As designed, $>70 \%$ of patients had ALCL, including $50 \%$ with $A L K$-negative and $22 \%$ with $A L K$ positive disease. The primary study endpoint was PFS, and the investigational arm had more than double the PFS of the control arm with CHOP (48 vs 20 months). That PFS benefit led to an OS benefit, with a significant reduction in risk of death for patients receiving $\mathrm{BV}+\mathrm{CHP}$ compared with $\mathrm{CHOP}$ alone.

"Median OS was not met for either arm of the study at the initial presentation," said Dr. Horwitz. "This is not a situation where CHOP is doing poorly; the investigational arm is truly better." Subgroup analysis showed a clear benefit in PFS and OS in patients with ALCL (both $A L K$-positive and -negative). "BV $+\mathrm{CHP}$ is a standard of care in the United States for CD30 expressing PTCL and is generally recommended for all adult patients with ALCL, both ALK-negative and $A L K$-positive disease," said Dr. Horwitz. "Although the study did not address consolidation with transplant, some data suggest that it may still improve outcomes, but it's hard to draw clear conclusions."

According to Dr. Horwitz, interpretation of the data outside of ALCL is more complicated. Patients with PTCL-NOS or AITL with CD30 expression were part of the intent-to-treat group in ECHELON-2, which showed PFS and OS benefit. However, "the subset size precludes statistical conclusions, so there is a lot of room for individual physicians to choose how to interpret these data," said Dr. Horwitz, who noted that no regimen has demonstrated unequivocal superiority to CHOP in this population. "If one wants to use something other than CHOP for patients with CD30-positive disease, we could treat patients with $\mathrm{BV}$ and chemotherapy, and I would still follow-up with autoSCT in CR1," he continued. "If clinicians choose not to use BV, the addition of etoposide to CHOP, followed by autoSCT, also may be better than CHOP alone."
For patients with CD30-negative disease treated offstudy, Dr. Horwitz suggested the same regimen along with enrollment in a clinical trial if available.

\section{Subtypes by Therapeutic Vulnerabilities}

According to Dr. Horwitz, clinicians are beginning to understand that different T-cell lymphoma subtypes may benefit from more specific therapies. A number of studies have shown that patients with AITL or PTCL with T-follicular helper phenotype are characterized by recurrent mutations in genes governing epigenetic functions, such as TET2, DNMT3A, or IDH2. A retrospective analysis has demonstrated higher rates of response, more frequent response, and more frequent complete responses to histone-deacetylase inhibition with $\mathrm{T}$-follicular helper subtypes, suggesting that it may be a better therapeutic approach for these diseases. ${ }^{4}$

A small study in France demonstrated impressive activity with azacytidine in patients with AITL, with 9 of 12 experiencing response, including complete and durable responses. ${ }^{5}$ Conversely, there were almost no responses in the non-AITL, PTCL-NOS subgroup.

Clinicians are also awaiting data from a large phase II study that randomized patients to romidepsin $+\mathrm{CHOP}$ or CHOP alone. ${ }^{6}$ According to Dr. Horwitz, this study will show whether romidepsin adds to CHOP in overall in T-cell lymphoma or perhaps in the AITL or follicular helper T-cell subset. Other targets being investigated include inhibitors of the JAK/STAT and PI3K pathways in T-cell lymphomas. An expansion cohort of a phase I study showed a $50 \%$ response rate in a small number of patients with PTCL treated with duvelisib, a PI3K-delta and PI3K-gamma inhibitor. ${ }^{7}$

A study of ruxolitinib, a JAK1/2 inhibitor, showed clinical benefit in patients with PTCL with either activating mutations in the JAK/STAT pathway or functional evidence of elevated pSTAT-3 and/or pSTAT- $5 .{ }^{8}$ Cerdulatinib, a SYK/ JAK1 and JAK2 inhibitor in development, also demonstrated an overall response rate $>50 \%$, said Dr. Horwitz, but these responses were almost all in patients with AITL or follicular helper T-cell lymphomas. ${ }^{9}$ There were responses in some rare T-cell lymphomas, such as gamma-delta T-cell lymphomas, but no responses in patients with PTCL-NOS.

Finally, tipifarnib, a farnesyltransferase inhibitor, has been shown to have activity in T-cell lymphoma, primarily in patients with AITL. ${ }^{10}$ Responders were greatly enriched for AITL patients with specific variants of KIR3DL2, said Dr. Horwitz.

A proposed Alliance/Intergroup trial will randomize patients with untreated T-cell lymphoma to 1 of 3 arms: standard therapy; duvelisib + CHOP \pm etoposide; or azacitidine $+\mathrm{CHOP} \pm$ etoposide. "The goal is to recapitulate what we saw on ECHELON-2 and improve results again by adding active agents to a chemotherapy backbone, 
and hopefully show benefit for a large subset of patients with T-cell lymphoma," Dr. Horwitz concluded.

Disclosures: Dr. Horwitz had disclosed that he has received consulting fees from Astex Pharmaceuticals; Celgene Corporation; Janssen Pharmaceutica Products, LP; Kura Oncology, Inc.; Kyowa Hakko Kirin Co., Ltd.; ADCT; C4
Therapeutics; Curio; Myeloid Therapeutics; Verastem; Seattle Genetics, Inc. and Takeda Pharmaceuticals North America, Inc. He has also received grant/ research support from Celgene Corporation; Daiichi-Sankyo Co.; Forty Seven Inc.; ADCT; Aileron; Corvus; Trillium; Verastem; Portola Pharmaceuticals, Inc. and Seattle Genetics, Inc.

Correspondence: Steven M. Horwitz, MD, Memorial Sloan Kettering Cancer Center, Department of Hematology, 1275 York Avenue, New York, NY 10021. Email: horwitzs@mskcc.org

\section{References}

1. Schmitz N, Trümper L, Ziepert M, et al. Treatment and prognosis of mature T-cell and NK-cell lymphoma: an analysis of patients with T-cell lymphoma treated in studies of the German High-Grade Non-Hodgkin Lymphoma Study Group. Blood 2010;116:3418-3425.

2. d'Amore F, Relander T, Lauritzsen GF, et al. Up-front autologous stem-cell transplantation in peripheral T-cell lymphoma: NLG-T-01. J Clin Oncol 2012;30:3093-3099.

3. Horwitz $\mathrm{S}, \mathrm{O}^{\prime}$ Connor OA, Pro B, et al. Brentuximab vedotin with chemotherapy for CD30-positive peripheral T-cell lymphoma (ECHELON-2): a global, double-blind, randomised, phase 3 trial. Lancet 2019;393: 229-240.

4. Ghione P, Ozkaya N, Faruque $\mathrm{P}$, et al. Romidepsin activity in T follicular helper (TFH)-phenotype PTCL versus non TFH treated on the same clinical trials [abstract]. J Clin Oncol 2018;36(Suppl):Abstract 7509.

5. Odejide $\mathrm{O}$, Weigert $\mathrm{O}$, Lane $\mathrm{AA}$, et al. A targeted mutational landscape of angioimmunoblastic T-cell lymphoma. Blood 2014;123:1293-1296.
6. Dupuis J, Morschhauser F, Ghesquières $\mathrm{H}$, et al. Combination of romidepsin with cyclophosphamide, doxorubicin, vincristine, and prednisone in previously untreated patients with peripheral T-cell lymphoma: a non-randomised, phase 1b/2 study. Lancet Haematol 2015;2:e160-165.

7. Horwitz SM, Koch R, Porcu P, et al. Activity of the PI3K- $\delta, \gamma$ inhibitor duvelisib in a phase 1 trial and preclinical models of T-cell lymphoma. Blood 2018:131:888-898.

8. Moskowitz AJ, Ghione P, Jacobsen ED, et al. Final results of a phase II biomarker-driven study of ruxolitinib in relapsed and refractory T-cell lymphoma [abstract]. Blood 2019;134(Suppl 1):Abstract 4019.

9. Ishikawa C, Senba M, Mori N. Anti-adult T-cell leukemia/lymphoma activity of cerdulatinib, a dual SYK/JAK kinase inhibitor. Int J Oncol 2018;53:1681-1690.

10. Witzig TE, Sokol L, Foss FM, et al. Proof of concept for tipifarnib in relapsed or refractory angioimmunoblastic T-cell lymphoma (AITL) and CXCL12+ peripheral T-cell lymphoma (PTCL): preliminary results from an open-label, phase 2 study [abstract]. Blood 2019;134(Suppl 1):Abstract 468. 